\title{
Stable Algorithm Based on Lax-Friedrichs Scheme for Visualization of Shallow Water
}

\author{
Bandung Arry Sanjoyo, Mochamad Hariadi, Mauridhi Hery Purnomo \\ Department of Electrical Engineering, Institut Teknologi Sepuluh Nopember, \\ Surabaya, Indonesia. \\ e-mail: bandung@matematika.its.ac.id, mochar@ee.its.ac.id, \\ hery@ee.its.ac.id
}

Received February 3, 2020; Revised March 26, 2020; Accepted May 11, 2020

\begin{abstract}
Many game applications require fluid flow visualization of shallow water, especially dam-break flow. A Shallow Water Equation (SWE) is a mathematical model of shallow water flow which can be used to compute the flow depth and velocity. We propose a stable algorithm for visualization of dam-break flow on flat and flat with bumps topography. We choose Lax-Friedrichs scheme as the numerical method for solving the SWE. Then, we investigate the consistency, stability, and convergence of the scheme. Finally, we transform the strategy into a visualization algorithm of SWE and analyze the complexity. The results of this paper are: 1) the Lax-Friedrichs scheme that is consistent and conditionally stable; furthermore, if the stability condition is satisfied, the scheme is convergent; 2) an algorithm to visualize flow depth and velocity which has complexity $O(N)$ in each time iteration. We have applied the algorithm to flat and flat with bumps topography. According to visualization results, the numerical solution is very close to analytical solution in the case of flat topography. In the case of flat with bumps topography, the algorithm can visualize the dam-break flow and after a long time the numerical solution is very close to the analytical steady-state solution. Hence the proposed visualization algorithm is suitable for game applications containing flat with bumps environments.
\end{abstract}

Keywords: shallow water equations, dam-break flow, Lax-Friedrichs scheme, flow visualization.

\section{INTRODUCTION}

Serious game application for flood requires an accurate information about flow depth and velocity to predict the distribution and to prevent flood [1]. Thus, the visualization of fluid flow is essentially needed in flood serious game application. However, visual movement of water has not been convincingly implemented. This is because many numerical methods are unstable for a long time and requires a long computation time. Therefore, we 
require a stable method for visualization of fluid flow. The goal of this work is to investigate a simple method for visualization of fluid flow, to be used in game application or as component objects in game development software. In this work, we focus on the stability of the algorithm rather than the order of accuracy.

In order to obtain the physical parameters of the flow, it is commonly used to formulate the flow into the Navier-Stokes partial differential equation system model, and its derivatives such as a Shallow Water Equations (SWE) and Saint-Vernant equations. The SWE have been widely used to conduct analysis, simulation, and visualization of open fluid flow. Recently, the SWE has been used to model many problems, such as tsunami [2]-[4], river flow [5], [6], and dam-break flow [7]-[9]. Many numerical methods such as finite difference [9]-[12], finite volume [13]-[15], and finite element can be used to solve the SWE systems. Lax-Friedrichs, Lax-Wendroff, Leap-Frog scheme are the three most popular methods in finite difference to find the solution of partial differential equations numerically [11], [12], [16]. In the case of the computation cost, we investigate the three schemes and select the fewest number of works done. Therefore, we focus on analyzing the reliability of the selected scheme for solving SWE systems, specifically looking for complexity, consistency, stability, and convergence. Furthermore, we design the algorithm based on the selected scheme to visualize the fluid flow. As case study, we test the program implementation of the algorithm on the shallow dam-break flow environment.

\section{RELATED WORKS}

The flow visualization can be done by using Computational Fluid Dynamics (CFD) based on Navier-Stokes equations. In creating fluid-like animation, three-dimensional Navier-Stokes has been developed to a new physics-based model to animate the rotational of gaseous motion [16]-[18]. Their visual simulation is based on a finite difference method and an explicit time solver. The explicit solvers have a problem for large time, i.e., the numerical scheme can become unstable. Jos Stam also simulates the fluid using Lagrangian and implicit methods to solve Navier-Stokes equations [19]. However, their cost of computation is expensive. Kellomäki simulated a large scale of water in games using various methods, one of the methods is SWE [20].

In the case of dam-break flow, the SWE has been widely used to visualize the fluid flow [2], [6], [9], [21]-[24]. There are many popular meshbased methods for solving the SWE, i.e., a Lax-Friedrichs, a Lax-Wendroff, and a Leap-Frog scheme. Ray compared the three schemes applied for a linear advection equation, and he shows that the Lax-Wendroff scheme is more accurate than the Lax-Friedrichs and a Leap-Frog scheme [25]. Mungkasi and Sari solved the SWE for flat topography and tested on a dam-break flow environment [26]. They compared three methods, i.e., the implicit collocated finite difference method, the explicit collocated Lax-Friedrichs finite volume 
method, and the explicit staggered finite volume. The Lax-Friedrichs finite volume method is stable according to the simulation results and simple to compute in terms of implementation of the algorithms. However, they did not compare the computation time of the three methods. Furthermore, they also did not study the consistency and stability of the three methods.

\section{ORIGINALITY}

Generally, the visualization of fluid flow in a game application has an arbitrary topography. Usually, the topography is not flat. In this research, we focus on flat and bumps topography. We proposed a stable algorithm for visualization of shallow water equations. The strategies of the algorithm design are choosing the simpler numerical scheme to solve the SWE, investigate the consistency, stability, and convergence of the scheme for SWE, and transform the strategy into an algorithm. The outputs of the algorithm are the depth and velocity of fluid flow. The depth of the flow is depicted in the visual movement of the flow every time. The specificity of this algorithm is to prioritize the stability and speed of the computation process rather than the accuracy of the results. Consequently, this algorithm can be used in visualizing fluid flow in serious game applications.

\section{PROPOSED METHOD}

We describe the proposed method in the following steps:

1) Analytical study In this step, we investigate the mathematical model of dam-break flow. Then, we study the consistency, stability, and convergence of Lax-Friedrichs scheme analytically.

2) Design and Analysis of Visualization Algorithm for SWE Based on the analytical study, we design a visualization algorithm for SWE. Then, we analyze the time complexity of the algorithm.

3) Implementation and Test Case We implement the visualization algorithm for SWE in Matlab. Then, we test the implementation program for some test cases. The test cases are dam-break flow where the topography is flat and flat with bumps.

\section{Mathematical Model of Dam-Break Flow}

The mathematical model of fluid flow is governed by a fluid flow model, which is called a Navier-Stokes equation [11]. In the case of shallow water, the one-dimensional Navier-Stokes equations are transformed into onedimensional SWE as a state in the system of equations (1) [12]. The first component in system of Equations (1) is called the continuum equation and the second is called the momentum equation.

$$
\frac{\partial h}{\partial t}=-\frac{\partial q}{\partial x}
$$




$$
\frac{\partial q}{\partial t}=-\frac{\partial q u}{\partial x}-\frac{1}{2} g \frac{\partial h^{2}}{\partial x}+g h \frac{\partial H}{\partial x}
$$

where $t \geq 0$ is time variable in second (sec), $x$ is horizontal space variable in meter $(m)$ and $x$ is an element of flow region $[0, L] \subset R, h=h(t, x)$ denote the water flow depth in meter $(m), q=q(t, x)=h u$ is a debit of flow in $m^{2} / \mathrm{sec}$, $u=u(t, x)$ is a velocity of flow in $x$-direction in $m / \mathrm{sec}, \frac{\partial}{\partial t}$ is first parsial derivative with respect to $t, \frac{\partial}{\partial x}$ is first partial derivative with respect to $x, g$ is a gravitational constant in $\mathrm{m} / \mathrm{sec}^{2}$, and $H=H(t, x)$ is the height of river bed. We assume that there is no bed erosion and flow sedimentation, so the $H=H(t, x)$ becomes $H=H(x)$ and $\frac{\partial H}{\partial x}$ becomes $\frac{d H}{d x}$. We assume that there is no friction in the riverbed. The Jacobian of (1) has characteristic roots $\lambda_{12}=u \pm \sqrt{g h}$.

The initial conditions of the SWE systems are supposed to be $u(0, x)=0$, and water flow depth is described in (2).

$$
h(0, x)=\left\{\begin{array}{l}
h_{l} \text { if } 0 \leq x \leq x_{d}, \\
h_{r} \text { if } x_{d} \leq x \leq L \text { and } h_{l}>h_{r}
\end{array}\right.
$$

When the flow is in a steady-state condition, the depth and the velocity of flow are unchanged in time $t$. So that system of equations (1) becomes Equation (3) and (4).

$$
\begin{aligned}
& \frac{\partial q}{\partial x}=0 \\
& \frac{\partial H}{\partial x}=\left(\frac{q^{2}}{g h}-1\right) \frac{\partial h}{\partial x}
\end{aligned}
$$

From equation (3), it means that the debit of flow is constant $q_{0}$. If we integrate Equation (4), then we obtain an analytical solution of the flow model in steady state as in Equation (5) or (6).

$$
\begin{aligned}
& \int_{0}^{H(x)} \frac{\partial H}{\partial x}=\int_{0}^{H(x)}\left(\frac{q^{2}}{g h}-1\right) \frac{\partial h}{\partial x} \\
& H(x)=\left(-\frac{q_{0}^{2}}{2 g h^{2}(x)}-h(x)\right)-\left(-\frac{q_{0}^{2}}{2 g h_{0}^{2}}-h_{0}\right)
\end{aligned}
$$

By arranging Equation (6) algebraically, we got cubic polynomial equation in $h(x)$ as in Equation (7).

$$
h^{3}(x)+\left(H(x)-\frac{q_{0}^{2}}{2 g h_{0}^{2}}-h_{0}\right) h^{2}(x)+\frac{q_{0}^{2}}{2 g}=0
$$


Equation (7) can be solved to obtain the depth of flow at each location, $h(x)$. We used Equation (7) to verify the computational results of the methods which is used to solve the SWE.

If we assume that there is no debit of flow comes into and goes from the systems, or $q_{0}=0$, then we obtain a lake-at-rest conditions or can be stated as in Equation (8).

$$
H(x)+h(x)=\text { Const }
$$

There are many analytical solutions for dam-break flow, including by Dressler [13], Stoker [14], and Ritter [15]. When the dam break is instantaneous, the bottom is flat, and there is no friction, the analytical solutions for $h(t, x)$ and $u(t, x)$ are as follows [16].

$$
\begin{aligned}
& h(t, x)= \begin{cases}h_{l} & \text { if } x \leq x_{A}(t), \\
\frac{4}{9 g}\left(\sqrt{g h_{l}}-\frac{x-x_{0}}{2 t}\right)^{2} & \text { if } \quad x_{A}(t) \leq x \leq x_{B}(t), \\
\frac{c_{m}{ }^{2}}{g} & \text { if } \quad x_{B}(t) \leq x \leq x_{C}(t), \\
h_{r} & \text { if } x_{C}(t) \leq x\end{cases} \\
& u(t, x)=\left\{\begin{array}{cll}
0 & \text { if } \quad x \leq x_{A}(t), \\
\frac{2}{3}\left(\frac{x-x_{0}}{t}+\sqrt{g h_{l}}\right) & \text { if } \quad x_{A}(t) \leq x \leq x_{B}(t), \\
2\left(\sqrt{g h_{l}}-c_{m}\right) & \text { if } \quad x_{B}(t) \leq x \leq x_{C}(t), \\
0 & \text { if } \quad x_{C}(t) \leq x
\end{array}\right.
\end{aligned}
$$

where $x_{A}(t)=x_{0}-t \sqrt{g h_{l}}$,

$$
\begin{aligned}
& x_{B}(t)=x_{0}+t\left(2 \sqrt{g h_{l}}-3 c_{m}\right), \text { and } \\
& x_{C}(t)=x_{0}+t\left(\frac{2 c_{m}^{2}\left(\sqrt{g h_{l}}-c_{m}\right)}{c_{m}^{2}-g h_{r}}\right)
\end{aligned}
$$

The parameter $c_{m}=\sqrt{g h_{m}}$ is a solution of (11).

$$
-8 g h_{r} c_{m}^{2}\left(g h_{l}-c_{m}^{2}\right)^{2}+\left(c_{m}^{2}-g h_{r}\right)^{2}\left(c_{m}^{2}+g h_{r}\right)^{2}=0
$$

\section{Computational Strategy and Lax-Friedrichs Scheme}

To find the solution of SWE system (1), we discretized the domain variable $t$ and $x$. We discretized time variable $t$ into $t_{0}, t_{1}, \ldots, t_{M}$, where $t_{M}$ is the duration of time simulation. We set $\Delta t_{i}=t_{i}-t_{i-1}$ for $i=1,2, \ldots, M$, so we obtain statement (12).

$$
\forall k \in Z^{+}, t_{k}=t_{0}+\sum_{i=1}^{k} \Delta t_{i}
$$


The space variable $x$ is discretized into $N$ equispaced of $\Delta x=L / N$, or we have $N+1$ nodes $x_{0}, x_{1}, \ldots, x_{N}$ where $x_{n}=n \Delta x$ for $n=0,1, \ldots, N$. Lets $U_{i}^{k}=\left(h_{i}^{k} \quad q_{i}^{k}\right)^{T}$ is the solution of system of equations (1) at node $x_{i}$ and at time $t_{k}$.

We designed the flow visualization displayed at each time $t_{k}$. Therefore, we choose forward difference discretization scheme in $t$ variable. The forward difference scheme has linear order of accuracy, $O(\Delta t)$. In order to obtain a more accurate solution, we choose centered difference discretization scheme in $x$ variable. The centered difference scheme has quadratic order of accuracy, $O\left(\Delta x^{2}\right)$. So, the order of accuracy of the forward in time and centered in space scheme is $O\left(\Delta x^{2}, \Delta t\right)$. The solution of systems of equation (1) using this scheme is unstable for large value of $t$. To stabilize this scheme, Lax-Friedrichs modified this scheme by defining $U_{i}^{k}$ as the average of $U_{i-1}^{k}$ and $U_{i+1}^{k}$. Applying this Lax-Friedrichs scheme to system of equations (1), we obtain a system of discrete Equations (13). The initial condition of (13) is $U_{i}^{0}=\left(\begin{array}{ll}h_{i}^{0} & q_{i}^{0}\end{array}\right)^{T}$.

$$
\begin{aligned}
& h_{i}^{k+1}=\frac{1}{2}\left(h_{i-1}^{k}+h_{i+1}^{k}\right)-\Delta t\left(\frac{\partial q}{\partial x}\right)_{i}^{k} \\
& q_{i}^{k+1}=\frac{1}{2}\left(q_{i-1}^{k}+q_{i+1}^{k}\right)-\Delta t\left(\frac{\partial G}{\partial x}\right)_{i}^{k}
\end{aligned}
$$

where $G=-q u-\frac{1}{2} g h^{2}+g h H$,

$$
\begin{aligned}
& \left(\frac{\partial q}{\partial x}\right)_{i}^{k}=\frac{1}{2 \Delta x}\left(q_{i+1}^{k}+q_{i-1}^{k}\right), \text { and } \\
& \left(\frac{\partial G}{\partial x}\right)_{i}^{k}=\frac{1}{2 \Delta x}\left(G_{i+1}^{k}+G_{i-1}^{k}\right) .
\end{aligned}
$$

The numerical solution $U_{i}^{k}$ can be found by iterating Equation (13). Each time step $k+1$, we obtain the solution $U_{i}^{k+1}=\left(h_{i}^{k+1} \quad q_{i}^{k+1}\right)^{T}$ and the number of work done is $O(N)$. The flow velocities $u_{i}^{k+1}$ could be computed using (14).

$$
u_{i}^{k+1}=\frac{q_{i}^{k+1}}{h_{i}^{k+1}}
$$

As we see in (14), if the depth of flow near zero, then we will get a numerical problem for the calculation of $u_{i}^{k+1}$.

\section{Consistency and Stability of Lax-Friedrichs Scheme for SWE}

A scheme for a system is consistent if the truncation error $\left(e_{T}\right)$ tends to zero when $\Delta t \rightarrow 0$ and $\Delta x \rightarrow 0$. A truncation error is a difference between a numerical scheme and its differential equation. So, we should check the truncation error of 
the continuum equation and the momentum equation. This paper only explains the truncation error for the continuum equations, while the truncation error for the momentum equations could be done in the same way.

Firstly, we expand the flow depth $h_{i}^{k}$ and the debit of flow $q_{i}^{k}$ using Taylor series as in equations (15)-(19).

$$
\begin{aligned}
& h_{i}^{k+1}-h_{i}^{k}=\left.\Delta t \frac{\partial h}{\partial t}\right|_{i} ^{k}+\left.\frac{\Delta t^{2}}{2} \frac{\partial^{2} h}{\partial t^{2}}\right|_{i} ^{k}+\left.\frac{\Delta t^{3}}{6} \frac{\partial^{3} h}{\partial t^{3}}\right|_{i} ^{k}+\ldots \\
& h_{i+1}^{k}-h_{i}^{k}=\left.\Delta x \frac{\partial h}{\partial x}\right|_{i} ^{k}+\left.\frac{\Delta x^{2}}{2} \frac{\partial^{2} h}{\partial x^{2}}\right|_{i} ^{k}+\left.\frac{\Delta x^{3}}{6} \frac{\partial^{3} h}{\partial x^{3}}\right|_{i} ^{k}+\ldots \\
& h_{i-1}^{k}-h_{i}^{k}=-\left.\Delta x \frac{\partial h}{\partial x}\right|_{i} ^{k}+\left.\frac{\Delta x^{2}}{2} \frac{\partial^{2} h}{\partial x^{2}}\right|_{i} ^{k}-\left.\frac{\Delta x^{3}}{6} \frac{\partial^{3} h}{\partial x^{3}}\right|_{i} ^{k}+\ldots \\
& q_{i+1}^{k}-q_{i}^{k}=\left.\Delta x \frac{\partial q}{\partial x}\right|_{i} ^{k}+\left.\frac{\Delta x^{2}}{2} \frac{\partial^{2} q}{\partial x^{2}}\right|_{i} ^{k}+\left.\frac{\Delta x^{3}}{6} \frac{\partial^{3} q}{\partial x^{3}}\right|_{i} ^{k}+\ldots \\
& q_{i-1}^{k}-q_{i}^{k}=-\left.\Delta x \frac{\partial q}{\partial x}\right|_{i} ^{k}+\left.\frac{\Delta x^{2}}{2} \frac{\partial^{2} q}{\partial x^{2}}\right|_{i} ^{k}-\left.\frac{\Delta x^{3}}{6} \frac{\partial^{3} q}{\partial x^{3}}\right|_{i} ^{k}+\ldots
\end{aligned}
$$

We combined the equations (15)-(19) and arranged the combined equations into a form (20).

$$
\left(h_{i}^{k+1}-\frac{1}{2}\left(h_{i+1}^{k}+h_{i-1}^{k}\right)+\frac{\Delta t}{2 \Delta x}\left(q_{i+1}^{k}-q_{i-1}^{k}\right)\right)-\left(\left.\frac{\partial h}{\partial t}\right|_{i} ^{k}+\left.\frac{\partial q}{\partial x}\right|_{i} ^{k}\right)=R_{h}
$$

where

$$
R_{h}=\Delta t\left(\left.\frac{\partial h}{\partial t}\right|_{i} ^{k}+\left.\frac{\partial q}{\partial x}\right|_{i} ^{k}\right)+\left.\frac{\Delta t^{2}}{2} \frac{\partial^{2} h}{\partial t^{2}}\right|_{i} ^{k}-\left.\frac{\Delta x^{2}}{2} \frac{\partial^{2} h}{\partial x^{2}}\right|_{i} ^{k}+\left.\frac{\Delta t^{3}}{6} \frac{\partial^{3} h}{\partial t^{3}}\right|_{i} ^{k}+\left.\Delta t \frac{\Delta x^{2}}{6} \frac{\partial^{3} h}{\partial x^{3}}\right|_{i} ^{k}+\ldots
$$

The first term of left-hand side of (20) is the numerical scheme of the continuum equation, and the second term is the continuum equation. Therefore, we took the truncation error of the numerical scheme of the continuum equation, as expressed in (21).

$$
e_{T}=\Delta t\left(\left.\frac{\partial h}{\partial t}\right|_{i} ^{k}+\left.\frac{\partial q}{\partial x}\right|_{i} ^{k}\right)+\left.\frac{\Delta t^{2}}{2} \frac{\partial^{2} h}{\partial t^{2}}\right|_{i} ^{k}-\left.\frac{\Delta x^{2}}{2} \frac{\partial^{2} h}{\partial x^{2}}\right|_{i} ^{k}+O\left(\Delta t^{3}, \Delta x^{2}\right)
$$

The limit of the truncation error as $\Delta x$ and $\Delta t$ tend to zero is zero, or the limit is expressed in (22). 


$$
\operatorname{Lim}_{\Delta x \rightarrow 0, \Delta t \rightarrow 0} e_{T}=0
$$

Equation (22) states that the Lax-Friedrichs scheme for the system of equations (1) is consistent.

Stability is a necessary and sufficient condition for convergence. In order to study the stability of the SWE, we use the linear advection eqation. That is because, the vectorized form of SWE (1) and linear advection equation is analogue. It is well known that the stability of the Lax-Friedrichs scheme for the linear advection equation will be achieved when the CFL is less than or equal to 1 , or $\sigma=c \frac{\Delta t}{\Delta x} \leq 1$ with $c$ is the speed of advection [17]. In the SWE, $c$ is $\max \left\{\left|\lambda_{1}\right|,\left|\lambda_{2}\right|\right\}$ and the CFL condition could be expressed as inequality (23).

$$
\frac{\Delta t_{i}}{\Delta x} \times \max _{1 \leq i \leq N}\left\{u_{i}+\sqrt{g h_{i}}|,| u_{i}-\sqrt{g h_{i}} \mid\right\} \leq 1
$$

So, the stability of the Lax-Friedrichs for SWE could be achieved when inequality (23) is satisfied. In other words, the Lax-Friedrichs scheme for SWE is conditionally stable. From equivalence theorem of Lax, the condition of convergence is consistency and stability. Therefore, if the condition in (23) is satisfied, then the Lax-Friedrichs for SWE is stable and convergent. In order to support this analytical result, we provide some computational experiments in the next section.

Inequality (23) leads to the strategy of taking the value of $\Delta t_{i}$, and we set the value of $\Delta t_{i}$ as expressed in (24).

$$
\Delta t_{i}=\frac{\Delta x}{\max _{1 \leq i \leq N}\left\{\left|u_{i}+\sqrt{g h_{i}}\right|,\left|u_{i}-\sqrt{g h_{i}}\right|\right.} \times S_{f}
$$

where $S_{f}$ is the safety factor. The value of $S_{f}$ could be set as a constant smaller than 1 but close to 1 .

\section{Algorithms of Flow Simulation}

Based on the above discussion, the algorithm for visualizing fluid flow can be designed as follows.

Input : Time of simulation $t_{0}$ and $\Delta t_{1}$, the height of topography $H_{0}$, and initial values of fluid flow parameters $h_{i}^{0}$ and $u_{i}^{0}$.

Output: Fluid flow parameters $h_{i}^{k}, q_{i}^{k}$ or $u_{i}^{k}$, and visualization of the fluid flow.

Algorithm:

1. Initialization step:

- Variable geometric grids, $\mathrm{H}(\mathrm{x})$

- Variable flow height $h(0, x)$, and flow velocity $u(0, x)$. 
2. For each time step do:

- Calculate $\Delta$ t using Equation (24)

- Calculate flow parameters $h_{i}^{k+1}, q_{i}^{k+1}, u_{i}^{k+1}$ using Equation (13) and (14)

- Apply the boundary conditions

- Visualize the flow variables $h_{i}^{k+1}$ and $u_{i}^{k+1}$.

End of time loop

As we see in the above algorithms, the number of works done in each time iteration is $O(N)$. Then, we implement the algorithm in Matlab, which will be used for experiments in the next section.

\section{EXPERIMENT AND ANALYSIS}

As mentioned in the Introduction, visualization of fluid is required in serious game applications. There are many physical environments (topography) in game applications. In this paper, we focus on dam-break flow where the topography is flat and flat with bumps. Such situations frequently occur in game applications. To verify the correctness of the visualization, we compare some numerical and analytical solutions in (8)-(11).

The first case is subcritical flow in dam-break flow on flat topography, or $H(x)=0$. The topography and the flow conditions are depicted in Figure 1 . The domain of flow is $x=[0, L]$, where $L=25 \mathrm{~m}$. We assumed that there is no fluid flow goes into and out of the boundary, or $q_{0}=0 \mathrm{~m}^{2} / \mathrm{sec}$ and $q_{L}=0 \mathrm{~m}^{2} / \mathrm{sec}$. The collapsed dam occurred after $t=0 \mathrm{sec}$ at location $x=5 \mathrm{~m}$. At the $t=0 \mathrm{sec}$, the depth of flow is expressed in (25) and the velocity at all location is $u(0, x)=0 \mathrm{~m} / \mathrm{sec}$.

$$
h(0, x)=\left\{\begin{array}{l}
5 \text { if } 0 \leq x \leq 5, \\
2_{r} \text { if } 0 \leq x \leq 25
\end{array}\right.
$$

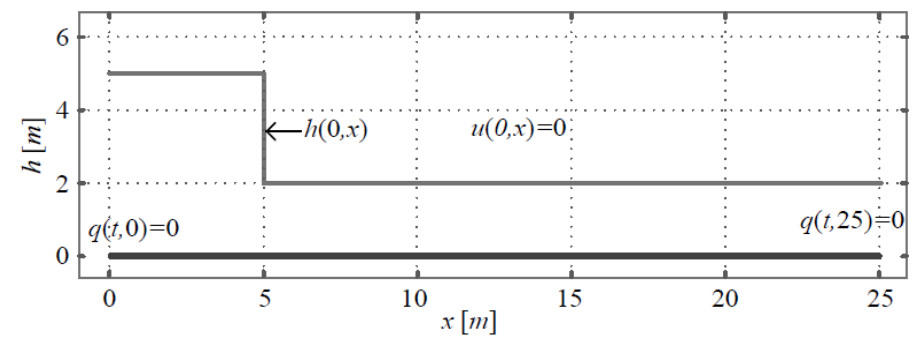

Figure 1. The topography and initial condition of flow in case 1.

The dam is collapsed at $t=0 \mathrm{sec}$. For $t>0 \mathrm{sec}$, the fluid flows towards the right side. The flow conditions at several values of $t$ are illustrated in Figure 2 . The numerical and the analytical solution for flow depth $h(t, x)$ have the same pattern. However, the flow velocity of numerical solution is slightly faster than the flow velocity of analytical solution. The summations 
$\operatorname{sum}\left(\left|h-h_{0}\right|\right) / N$ and $\operatorname{sum}\left(\left|u-u_{0}\right|\right) / N$ in Figure 2 express the average of the absolute difference between flow depth and flow velocity at $t$ seconds and at $(t+\Delta t)$ seconds. We found that as time $t$ increases, the average values decrease to zero. This means the condition of the flow is going to a steadystate condition. When $t \geq 15.56 \mathrm{sec}$, the flow depth is constant, and the flow velocity is zero. This condition shows that the flow is in a steady state. It is suitable with the Equation (8).
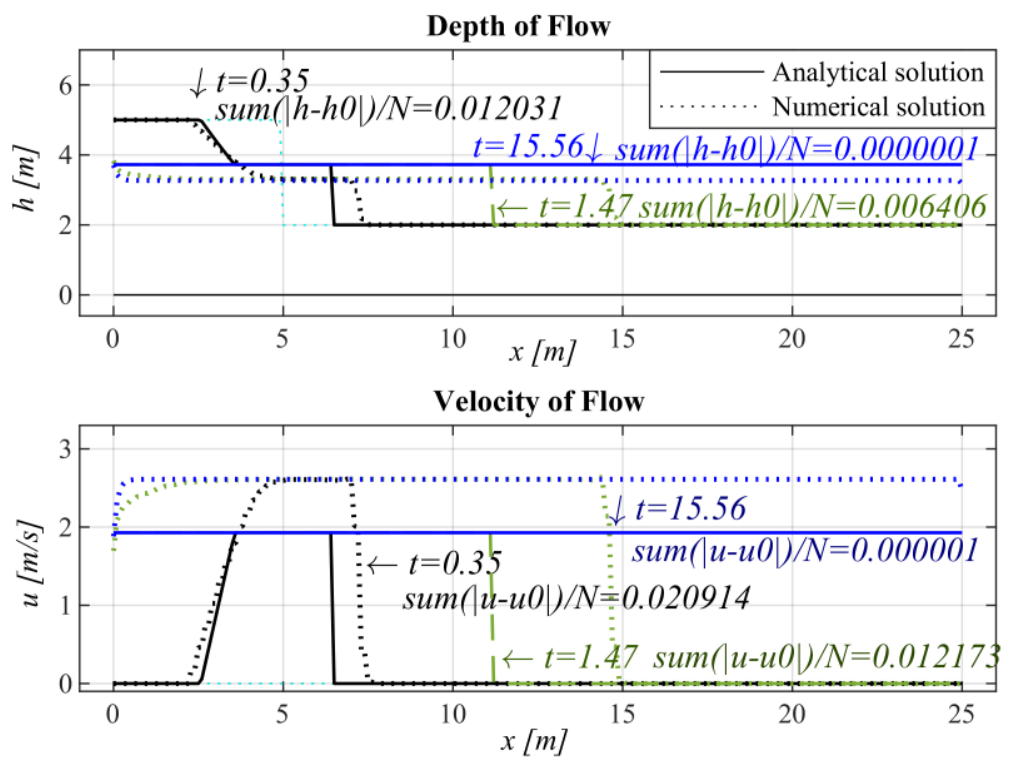

Figure 2. The profile of depth and velocity of flow beyond dam-break for flat topography.

The second case is the dam-break subcritical fluid flow on bumps topography. The initial conditions and the domain of fluid flow are the same with the previous case. However, the bottom topography of fluid flow $H(x)$ is the bumps. We set the height of the bump $H(x)$ as

$$
H(x)= \begin{cases}e^{(-x-10)^{2}} & \text { if } 7 \leq x<13, \\ 0 & \text { others }\end{cases}
$$

The collapsed dam occurred after $t=0 \mathrm{sec}$ at location $x=5 \mathrm{~m}$. There is no exact solution for this type of flow. The result of running program are shown in Figure 3. We only capture the visualization of flow at $t=0 \mathrm{sec}$, $t=12.87 \mathrm{sec}, t=1999.63 \mathrm{sec}$ and $t=2010.24 \mathrm{sec}$. During the visualization process, when time $t$ goes to large value the flow goes to steady state condition. This is suitable with the steady-state analytical solution in (8).

The next numerical investigation is about the convergency of the numerical solution. A numerical solution is said to be convergent if we set the $\Delta t$ and $\Delta x$ tends to zero, then the numerical solution must tend to the exact solution of the mathematical model. For this purpose, we defined the 
differences between the exact and numerical solution as an error $L_{1}$ as expressed in Equation (27).

$$
L_{1}=\frac{1}{N} \sum_{i=1}^{N}\left|u_{i}^{e}-u_{i}^{c}\right|
$$

where $u_{i}^{e}$ is the exact solution and $u_{i}^{c}$ is the numerical solution at each grid point $i$.

We investigate the error of $L_{1}$ for both flow depth and velocity. We therefore refer to Equation (27) to calculate the error of numerical computations. For the investigations, we use various grid number and run the program for large $t$, then we record the error $L_{1}$. The results are depicted in Figure 4.
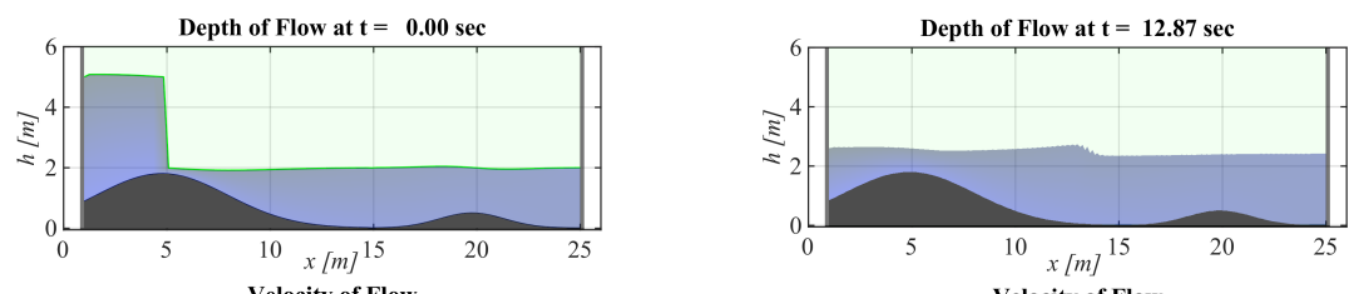

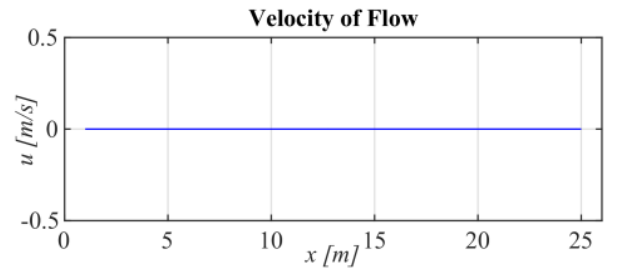

(a)
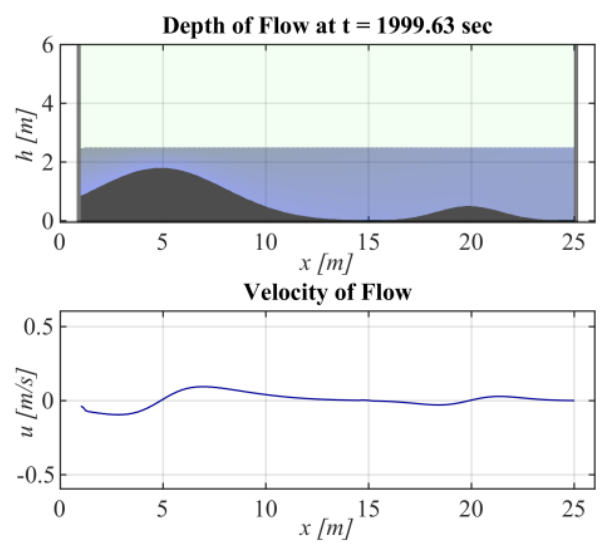

(c)

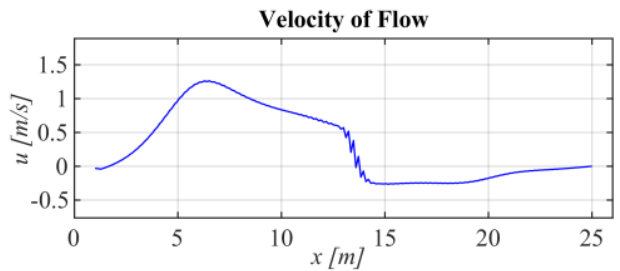

(b)
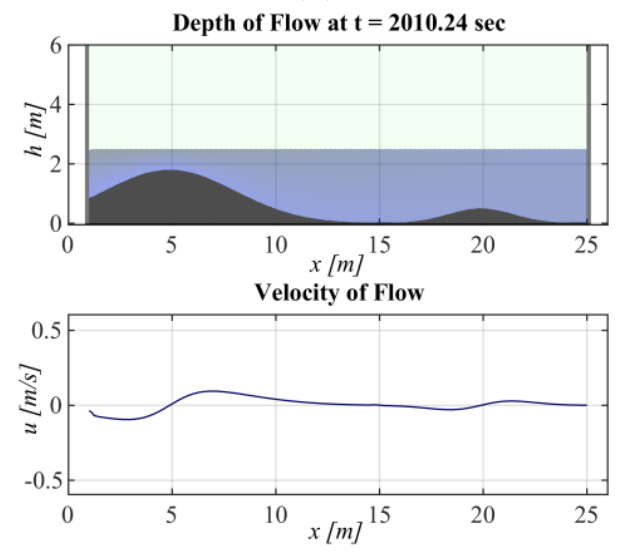

(d)

Figure 3. The visualization of fluid flow on bumps topography when time goes to large value.

Figure 4(a) displays the profile of error $L_{1}$ of the flow depth and velocity for $N=201$. Observe that starting from $t=20 \mathrm{sec}$, the error $L_{1}$ is almost constant and do not exceed a specific constant $K$. Thus, it satisfies the inequality (28). We also display the profile error $L_{1}$ for $N=301, N=401$, and 
$N=501$ in Figure 4(b), 4(c), and 4(d), respectively. The three cases also satisfy inequality (28).

$$
\operatorname{Lim}_{t \rightarrow \infty} \frac{1}{N} \sum_{i=1}^{N}\left|u_{i}^{e}-u_{i}^{c}\right| \leq K
$$

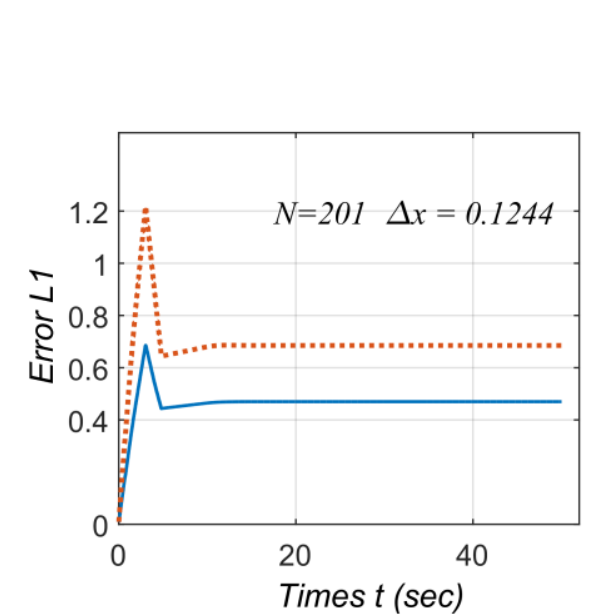

(a)

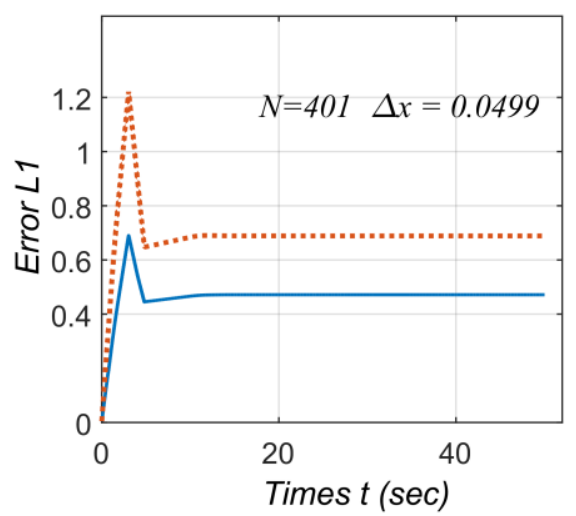

(c)

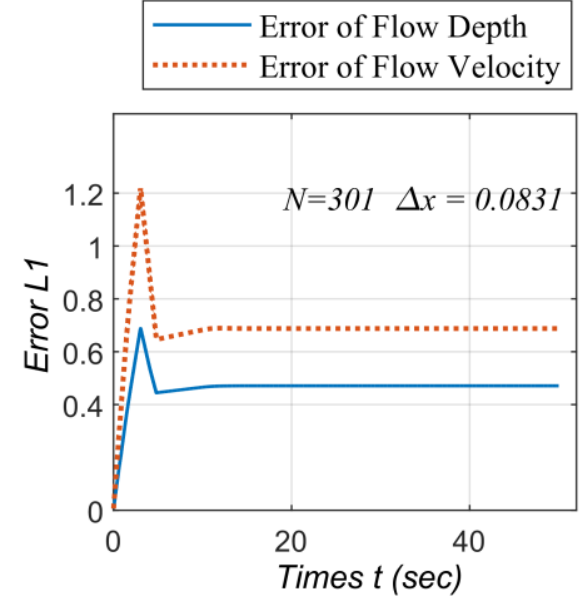

(b)

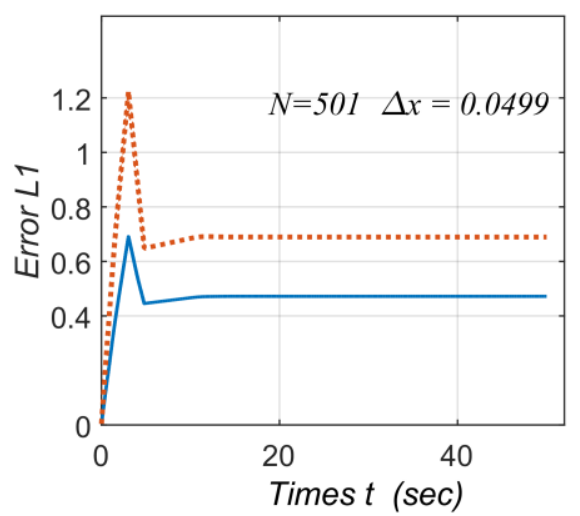

(d)

Figure 4. A Graph of error $L_{1}$ versus times $t$ for some grid numbers

Since the error $L_{1}$ is bounded, the Lax-Friedrichs scheme for the SWE is numerically stable. Further, when this scheme is applied to visualize the fluid flow for a longer time, then we guarantee that this scheme can run well, i.e., the error does not exceed some constant $K$. This shows that the LaxFriedrichs scheme for SWE is numericaly stable.

Figure 5 shows the relationships of norm error $L_{1}$ toward the variations of grid numbers for flow depth and velocity. The investigations are carried out for the number of grids from $N=201$ up to $N=601$. When the number of grids is higher, then the error of $L_{1}$ for flow depth and velocity become higher too. However, the propagation of the error is very small and tends to be constant. Thus, the Lax-Friedrichs scheme for SWE is numerically 
stable. Since we have shown that the method is consistent, the Lax-Friedrichs scheme for the SWE fluid flow problem on environment of dam-break flow is convergent.

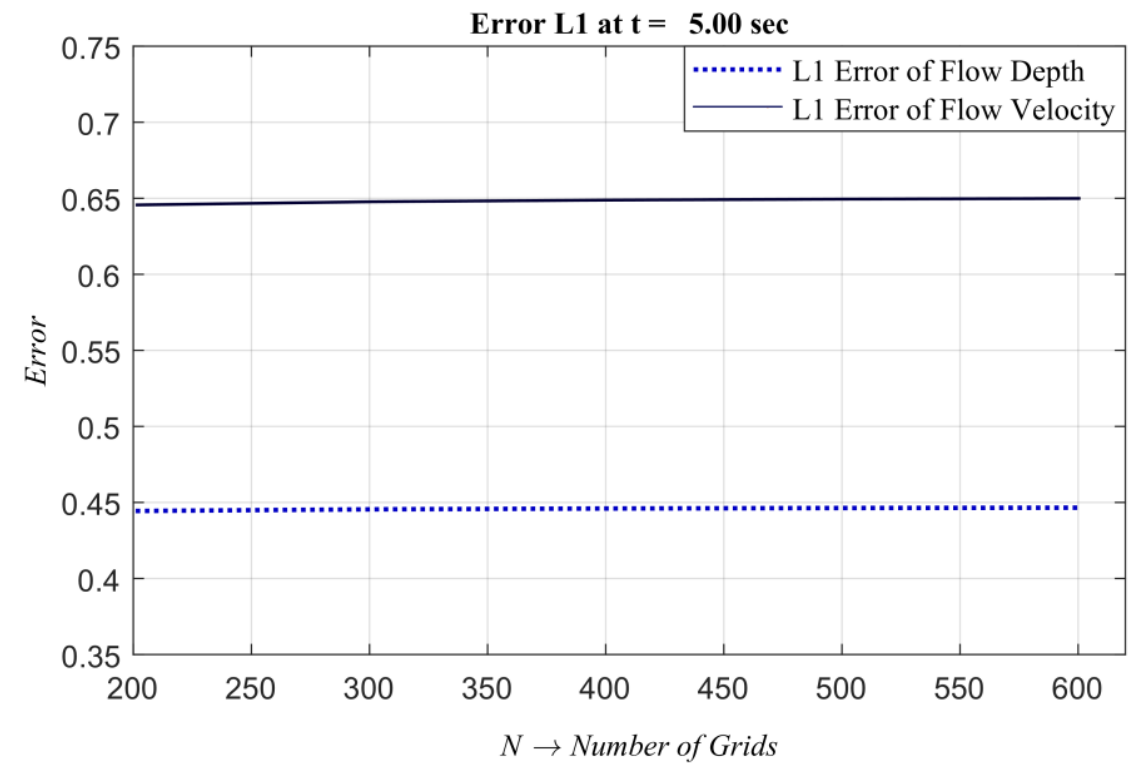

Figure 5. A graph of error $L_{1}$ versus number of grids

\section{CONCLUSION}

According to the results, we conclude that the algorithm for visualization of shallow water dam-break flow is as follows.

1. In each time step, the number of works done by the algorithm based on Lax-Friedrichs scheme is of order $O(N)$.

2. The Lax-Friedrichs scheme for SWE in dam-break flow is consistent and conditionally stable. If the stability condition is satisfied, the algorithm is convergent.

3. According to the visualization result:

- in the steady state case, the numerical solution is suitable with the analytical solution.

- In the case of flat topography, starting from $t=20 \mathrm{sec}$, the error is almost constant and do not exceed a specific constant $K$.

Hence, the algorithm is suitable for game applications with the environment containing flat and flat with bumps topography.

\section{Acknowledgements}

The authors would like to thank the Ministry of Research, Technology, and Higher Education of the Republic of Indonesia for providing the Postgraduate Education Scholarship for Domestic Affairs (BPPDN) to the author for the purpose of studying the Doctoral Program at the Department of Electrical Engineering ITS. 


\section{REFERENCES}

[1] M.-H. Tsai, Y.-L. Chang, J.-S. Shiau, and S.-M. Wang, Exploring the effects of a serious game-based learning package for disaster prevention education: The case of Battle of Flooding Protection, Int. J. Disaster Risk Reduct., vol. 43, p. 101393, Feb. 2020, doi: 10.1016/j.ijdrr.2019.101393.

[2] R. J. LeVeque, D. L. George, and M. J. Berger, Tsunami modelling with adaptively refined finite volume methods, Acta Numer., vol. 20, pp. 211-289, May 2011, doi: 10.1017/S0962492911000043.

[3] M. Zuhair and S. Alam, Tsunami Impacts on Nuclear Power Plants along Western Coast of India Due to a Great Makran Earthquake: A Numerical Simulation Approach, Int. J. Geosci., vol. 08, no. 12, pp. 1417-1426, 2017, doi: 10.4236/ijg.2017.812083.

[4] H. Altaie, Applications of a Nested Model for 2D Shallow Water equations in Tsunami Model, vol. 2, no. 3, p. 13, 2018.

[5] N. Gouta and F. Maurel, A finite volume solver for 1D shallow-water equations applied to an actual river, Int. J. Numer. Methods Fluids, vol. 38, no. 1, pp. 1-19, Jan. 2002, doi: 10.1002/fld.201.

[6] P. Glaister, An efficient numerical method for subcritical and supercritical open channel flows, Appl. Numer. Math., vol. 11, no. 6, pp. 497-508, Apr. 1993, doi: 10.1016/0168-9274(93)90089-A.

[7] O. Castro-Orgaz and H. Chanson, Ritter's dry-bed dam-break flows: positive and negative wave dynamics, Environ. Fluid Mech., vol. 17, no. 4, pp. 665-694, Aug. 2017, doi: 10.1007/s10652-017-9512-5.

[8] E. Chaabelasri, Numerical Simulation of Dam Break Flows Using a Radial Basis Function Meshless Method with Artificial Viscosity, Model. Simul. Eng., vol. 2018, pp. 1-11, 2018, doi: 10.1155/2018/4245658.

[9] A. Khoshkonesh, B. Nsom, S. Gohari, and H. Banejad, A comprehensive study on dam-break flow over dry and wet beds, Ocean Eng., vol. 188, p. 106279, Sep. 2019, doi: 10.1016/j.oceaneng.2019.106279.

[10] H. P. Gunawan, Numerical simulation of shallow water equations and related models, Institut Teknologi Bandung, L'Université Paris-Est, 2015.

[11] L. Rezzolla, Finite-difference Methods for the Solution of Partial Differential Equations, Frankfurt, 2018.

[12] N. D. Katopodes, Finite-Difference Methods for Advection, in FreeSurface Flow, Elsevier, 2019, pp. 118-197.

[13] S. Bi, J. Zhou, Y. Liu, and L. Song, A Finite Volume Method for Modeling Shallow Flows with Wet-Dry Fronts on Adaptive Cartesian Grids, Math. Probl. Eng., vol. 2014, Jul. 2014, doi: 10.1155/2014/209562.

[14] R. Touma and F. Kanbar, Well-balanced central schemes for twodimensional systems of shallow water equations with wet and dry 
states, Appl. Math. Model., vol. 62, pp. 728-750, Oct. 2018, doi: 10.1016/j.apm.2018.06.032.

[15] M. D. Thanh and N. X. Thanh, Well-Balanced Numerical Schemes for Shallow Water Equations with Horizontal Temperature Gradient, Bull. Malays. Math. Sci. Soc., vol. 43, no. 1, pp. 783-807, Jan. 2020, doi: 10.1007/s40840-018-00713-5.

[16] Michael Griebel, Thomas Dornseifer, and Tilman Neunhoeffer, Numerical Simulation in Fluid Dynamics: A Practical Introduction. Philadelphia: SIAM, 1998.

[17] N. Foster and D. Metaxas, Modeling the motion of a hot, turbulent gas, in Proceedings of the 24th annual conference on Computer graphics and interactive techniques - SIGGRAPH '97, Not Known, 1997, pp. 181-188, doi: $10.1145 / 258734.258838$.

[18] S. Nugroho and C. Citrahardhani, CFD Analysis of Nozzle Exit Position Effect in Ejector Gas Removal System in Geothermal Power Plant, Emit. Int. J. Eng. Technol., vol. 3, no. 1, Jun. 2015, doi: 10.24003/emitter.v3i1.35.

[19] J. Stam, Stable Fluids, in Siggraph, 1999, vol. 99, pp. 121-128.

[20] Kellomäki, T, Large-Scale Water Simulation in Games, Thesis for the degree of Doctor, Tampere University of Technology, Tampere, 2015.

[21] A. B. Almeida and A. B. Franco, Modeling of Dam-Break Flow, in Computer Modeling of Free-Surface and Pressurized Flows, M. H. Chaudhry and L. W. Mays, Eds. Dordrecht: Springer Netherlands, 1994, pp. 343-373.

[22] O. Delestre et al., SWASHES: a compilation of Shallow Water Analytic Solutions for Hydraulic and Environmental Studies. Wiley, Jan. 19, 2016.

[23] A. I. Aleksyuk and V. V. Belikov, The uniqueness of the exact solution of the Riemann problem for the shallow water equations with discontinuous bottom, J. Comput. Phys., vol. 390, pp. 232-248, Aug. 2019, doi: 10.1016/j.jcp.2019.04.001.

[24] S. Chen, B. Liao, and T. Huang, Corrected SPH methods for solving shallow-water equations, J. Hydrodyn., vol. 28, no. 3, pp. 389-399, Jun. 2016, doi: 10.1016/S1001-6058(16)60642-X.

[25] S. K. Ray, Comparison of Numerical Schemes for Shallow Water Equation, Glob. J. Inc USA, vol. 13, no. 4, p. 20, 2013.

[26] S. Mungkasi and I. P. Sari, Numerical solution to the shallow water equations using explicit and implicit schemes, presented at the THE 2016 Conference on Fundamental and Applied Science for Advanced Technology (CONFAST 2016): Proceeding of ConFAST 2016 Conference Series: International Conference on Physics and Applied Physics Research (ICPR 2016), International Conference on Industrial Biology (ICIBio 2016), and International Conference on Information System and Applied Mathematics (ICIAMath 2016), Yogyakarta, Indonesia, 2016, p. 020064, doi: 10.1063/1.4953989. 
[27] M. Griebel, T. Dornseifer, and T. Neunhoeffer, Numerical Simulation in Fluid Dynamics: A Practical Introduction. Philadelphia: SIAM, 1998.

[28] J. Sainte-Marie and M.-0. Bristeau, Derivation of a non-hydrostatic shallow water model; Comparison with Saint-Venant and Boussinesq systems, Discrete Contin. Dyn. Syst. - Ser. B, vol. 10, no. 4, pp. 733-759, Aug. 2008, doi: 10.3934/dcdsb.2008.10.733.

[29] Robert F. Dressler, Hydraulic resistance effect upon the dam-break functions., Journal of Research of the National Bureau of Standards, vol. 49, no. 3, pp. 217-225, Sep. 1952.

[30] J. J. Stoker, Water Waves The Mathematical Theory With Applications. Interscience Publishers, Inc., 1957. 